\title{
Estudios descoloniales del management y las organizaciones: avances, desafíos y prospectos
}

Jenny K. Rodríguez a

Universidad de Manchester, Reino Unido

jenny.rodriguez@manchester.ac.uk

Marcela Mandiola

Universidad Alberto Hurtado, Chile

ORCID: http://orcid.org/0000-0001-6604-985X

Hernán Camilo Pulido Martinez

Pontificia Universidad Javeriana, Colombia

ORCID: http://orcid.org/0000-0003-1071-2784

Alba Luz Giraldo Tamayo

Pontificia Universidad Javeriana, Colombia

ORCID: http://orcid.org/0000-0002-1409-8446
DOI: https://doi.org/10.11144/Javeriana.cao32-58.edmo Redalyc: http://www.redalyc.org/articulo.oa?id=20560207001

Fecha de recepción: 01 Marzo 2019

Fecha de aprobación: 29 Marzo 2019

Fecha de publicación: 30 Junio 2019

En el campo del management y las organizaciones, la producción y difusión dominantes del conocimiento han reproducido históricamente el pensamiento y las lógicas de los centros hegemónicos. Como resultado, prácticas esperadas y aceptadas replican interpretaciones y posturas epistémicas que asemejan las dinámicas de la colonialidad. Dicha colonialidad intelectual ha obscurecido e invisibilizado, por un lado, proyectos intelectuales alternativos; y por otro, ha presentado como universales principios reguladores de lo intelectual que son histórico, social, cultural y políticamente incompatibles con el pensamiento y las realidades de la periferia.

Este número especial de Cuadernos de Administración presenta discusiones que, desde una mirada latinoamericana y latinoamericanista, abordan el significado de lo crítico y la criticalidad en la producción de conocimiento acerca del management y las organizaciones. Los artículos que conforman este volumen de la revista dejan de lado las representaciones autoritarias que han sostenido ideas hegemónicas y utilizan como punto de partida el conocimiento producido en el margen. Esta comprensión de la criticalidad se fundamental en una postura decolonial (ver Grosfoguel, 2007), la cual utiliza nociones de ruptura en lugar de nociones de (re)negociación intelectual. Esto es, el desarrollo de una mirada epistemológicamente situada desde/ hacia lo crítico, buscando rechazar críticas domesticadas que re-negocien o re-formulen significados que finalmente expanden el conocimiento dominante. Así, este pensamiento "desde el margen” es el pensamiento hegemónico "del margen".

El valor y contribución de los trabajos presentados en este especial se centra en la manera en que, a través de diversas propuestas analíticas toma control del diálogo intelectual en formas que son representativas de la comunidad epistémica donde se posiciona y con la cual establece dialogo. Este es el pilar del proyecto decolonial, el cual se caracteriza por ser una desobediencia epistémica que quiebra el diálogo existente y lo re-inicia desde una posición que reconoce y utiliza la diversidad social, geopolítica e intelectual de la

Notas de autor: 
subalternidad, desafiando el reduccionismo intelectual y dando prioridad a las interpretaciones sostenidas por la ética y política pluriversal (ver Mignolo, 2011).

La propuesta intelectual de este número es una forma de respuesta al llamado a desafiar la colonialidad global, el etnocentrismo intelectual, el anglocentrismo hegemónico y la insularidad analítica (ver Quijano, 2009; Dussel, 2001; Bhambra, 2014). Decolonizar la producción y difusión del conocimiento en management y organizaciones no sólo legitima la validez intelectual del conocimiento desde los márgenes, en los márgenes y para los márgenes, sino que reconoce la personalidad intelectual tanto de quienes lo producen como de quienes lo reportan. Por ejemplo, los llamados estudios críticos del management -CMSse construyen sobre la Teoría Crítica de Frankfurt, a partir de la cual definen e identifican relaciones de control y poder. Esta postura analítica ha ganado legitimidad y se ha convertido en una crítica legitimada que continúa reproduciendo los patrones de colonialidad intelectual eurocéntrica.

Concretamente, los términos de referencia que tradicionalmente han sido utilizados por los enfoques críticos del management y las organizaciones utilizan dialécticas no reflexivas que minimizan el rol de las lógicas de pensamiento dominante y adoptan posturas más bien condescendientes, tales como la idea de 'dar voz al conocimiento y los hablantes marginalizados’. Asimismo, se sustentan en el diálogo entre interlocutores imperialistas, que en lugar de interrogar su posición privilegiada y opresiva como parte de la academia dominante, se enfocan en buscar soluciones a los problemas de inclusividad intelectual que identifican desde su perspectiva también dominante. En este sentido, la tradición de los estudios críticos del management continúa desarrollando formas de crítica domesticada que son articuladas dentro de los límites aceptables de la desconstrucción, pero que siguen reproduciendo jerarquías de exclusión o pseudo inclusión que sirven para legitimar su aparente postura auto-crítica y la democratización del debate.

Este número especial es, en cierta medida, una contra-respuesta a los estudios críticos del management pues resalta las posibilidades de rechazar la apropiación intelectual de sus principios y en su lugar presenta evidencia de las diversas formas en las cuales la postura decolonial ayuda a desarrollar el pensamiento teórico/ conceptual y empírico sobre management y organizaciones desde el margen.

El artículo que abre este volumen, tiene la autoría de Ana Paula Madeiros, Vanessa Faria Silva y Sergio Eduardo de Pinho Velho Wanderley, el cual presenta una geopolítica del conocimiento producido acerca de la descolonialidad. Los autores señalan que la propuesta constituida por los estudios acerca de la modenidad/ colonialidad/descolonialidad destacan la región latinoamericana, apartándose de las visiones universalistas que caracterizan los estudios postcoloniales. También señalan que muchos de los principales investigadores que han hecho aportes a este campo tienen vínculos con la academia anglosajona, o bien con la producción que se realiza en las universidades de los países hispanohablantes. El artículo reflexiona sobre el lugar que tiene la producción de conocimiento en portugués para los estudios organizacionales, resaltando que autores como Freire, Riberos y Santos, quienes se destacan en la literatura crítica, se han convertido en referentes constitutivos del campo en la perspectiva descolonial. El concepto de "border thinking" o pensamiento fronterizo, permite a los autores enfatizar la existencia de otros autores potenciales de la tradición crítica en Brasil, tales como Guerreiro Ramos y Celso Furtado, quienes podrían, o quizás deberían, ser incluidos para enriquecer tanto la forma de conducir la reflexión conceptual como la práctica investigativa. Un argumento importante de que antes de que se propusiera el "border thinking" como una herramienta conceptual potente para analizar las realidades latinoamericanas, elementos de esta forma de pensamiento ya estaban presentes en las obras de estos autores.

En el segundo artículo, Liliana Vargas Monroy introduce dos formas de pensamiento crítico útiles para el análisis de las realidades organizacionales propias de Latinoamérica. El artículo pone en dialogo los estudios sobre la gubernamentalidad con los estudios feministas decoloniales con el fin de analizar las transformaciones que han tenido lugar en uno de los sectores productivos más importantes en Colombia. En particular se presentan dos momentos en el desarrollo del sector de la flor cortada para mostrar como los 
cambios en la manera de "conducir la conducta" siguen las prescripciones para la implementación del modelo neoliberal que se instauraron en el sector.

El tercer artículo, escrito por Vicente Sisto, se concentra en el análisis del “managerialismo” y su vinculación con la expansión de las prescripciones neoliberales y la apropiación en los procesos de modernización en Chile. En el marco de esta discusión, el artículo explora prácticas alternativas de organización que se presentan en el escenario educativo chileno, las cuales van más allá de la impronta colonial que el "managerialismo" adquiere cuando se aplica en regiones distintas a las de origen. Las prácticas identificadas en la investigación permiten observar maneras de promover ciertas formas de vivir en conjunto que se apartan de la individualización concerniente a las formas de gobierno que requiere la expansión del modelo económico neoliberal, moviéndos hacia la construcción de modos de organización que se sustentan en la solidaridad y la cooperación.

En el cuarto artículo, Juan Manuel Arévalo Viveros, Franklyn Mosquera Pisso y Diego Cáceres Barajas discuten los problemas asociados al desarrollo y sus propuestas alternativas, denominadas bajo el rótulo de posdesarrollo. El artículo se aproxima a experiencias de organización en una región del sur-occidente colombiano para explorar los discursos que facilitan pensar alternativas posdesarrollistas que sirvan como guía para reconfigurar el mundo empresarial. Esta reconfiguración conlleva la intención crítica de superar el puesto que tiene el capital en las organizaciones contemporáneas y, a la vez, tiene como objetivo la búsqueda de otras ontologías y epistemologías "bajo las cuales se tenga otra forma de ver, saber y hacer en el mundo". Estas son presentadas como formas de ser y estar, alternativas a las imperantes asociadas a las prescripciones neoliberales. Una consideración importante en este trabajo es el valor que otorgan los autores a la recuperación histórica del territorio en donde se hacen presentes las organizaciones que se discuten en el artículo.

La fotografía como herramienta para explorar las realidades organizacionales que emergen en Brasil son utilizadas por Jaqueline Tittoni, Diego Drescher de Castro y Arthur Gomes de Almeida en el quinto artículo. Los estudios sobre la gubernamentalidad son nuevamente puestos al servicio de la exploración de la relación entre trabajo, conocimiento y la producción de la subjetividad. Los autores enfatizan el género y la raza como aspectos constitutivos de la subjetividad que se configuran para explorar las dimensiones coloniales asociadas al trabajo y a la producción de conocimiento acerca de grupos humanos específicos. Enseguida, exploran las posibilidades abiertas por los estudios que consideran las relaciones de poder, saber, subjetividad y colonialidad, sin dejar de reflexionar sobre las limitaciones que este marco tiene a la hora de considerar problemas particulares del mundo del trabajo en Latinoamérica. Estas limitaciones, argumentan, los movieron a explorar la fotografía como posibilidad para considerar el mundo del trabajo con una perspectiva decolonial que muestra visibilidades que son políticas, culturales, sociales y económicas, las cuales a su vez manifiestan subordinaciones, sometimientos y posibles líneas de fuga.

El sexto artículo, autoría de Oscar Andrés López Cortes, es el resultado de un proceso etnográfico llevado a cabo en el sector público en Colombia. El autor considera una serie de prescripciones que se deben implementar en la región como respuesta a las posiciones que adoptan contemporáneamente entidades reguladoras como la OCDE cuando realizan ejercicios de gobierno de manera internacional que configuran y reproducen relaciones de colonialidad. La aplicación de la lógica "managerial” al sector público es descrita a partir de casos particulares en donde se examina cómo entran en situaciones paradójicas la producción de sujeto con vocación y las prescripciones que buscan traer la lógica de la administración del sector privado a la administración pública.

En el séptimo artículo, Ángela Vera Ruiz, Cesar Andrés Llanco y Luis Alberto Guevara reflexionan sobre la formación de los psicólogos en el Perú, problematizando y explorando alternativas alrededor de estrategias para el análisis e intervención del rol en pequeñas y medianas empresas. Los autores relatan la experiencia particular con uno de los saberes coloniales que se ha difundido ampliamente para la administración de los ámbitos laborales. La psicología como herramienta para la administración de las organizaciones es examinada en términos de su lugar y sus operaciones, señalando la necesidad de construir otros tipos de conocimiento 
situados y críticos que sean epistémicamente consistentes con las situaciones que enfrentan las pequeñas y medianas empresas en la región.

El número especial cierra con la contribución de Maria Ceci Araujo Misoczky, quien realiza un análisis reflexivo acerca de los estudios organizacionales críticos. La autora examina los logros alcanzados, e identifica posibles caminos a seguir para el debate y la investigación. Retomando autores centrales que han sido utilizados para configurar el campo de los estudios con un corte crítico, el artículo reflexiona sobre los propios estudios de corte poscolonial y descolonial, mostrando sus divergencias para abrir así un análisis de perspectivas conceptuales que son, simultáneamente, cercanas y distantes cuando se trata de considerar tanto su lugar dentro del mundo académico, como su puesto en relación con la práctica y el activismo necesarios para guiar transformaciones o alternativas a las organizaciones y las formas de relacionarnos que el capitalismo ha naturalizado.

El trabajo intelectual presentado en este número especial de Cuadernos apunta a las posibilidades y necesidad de investigación y discusión teórica, conceptual y empírica que identifiquen, utilicen y desarrollen herramientas provistas dentro de los estudios descoloniales para analizar problemas de trabajo, management y organizaciones en la región latinoamericana y, de manera general, en lo que se considera el margen. Muy esquemáticamente habría que señalar que, si bien los artículos muestran evidencia de creatividad analítica en el uso de herramientas descoloniales y dan como resultado perspectivas críticas que anuncian posibles caminos para la transformación de las realidades organizacionales subordinadas, también se denota un cierto "atasco" en las formas en las cuales se confrontan los problemas que los autores consideran en sus investigaciones. Esto es, los trabajos muestran un manejo comprensivo del marco teórico-conceptual, pero la traducción de este marco para su uso empírico requiere mayor desarrollo y refinamiento.

En este sentido, el avance de estudios críticos decoloniales sobre management y organizaciones se beneficiaría de repensar no solo la forma de usar las herramientas descoloniales apropiadas, acondicionadas, adaptadas e hibridizadas, sino también el tipo de conocimiento que producen estos procesos. El cuerpo teórico-conceptual vinculado con el giro descolonial ha sido llevado rápidamente a diversos campos y disciplinas, entrando en contacto con los problemas, debates y objetos que tradicionalmente componen dichos campos y disciplinas y sus diversos límites y posibilidades. En este sentido, el futuro debe considerar las implicaciones que tiene esta traducción de un campo a otro y quizás articular y desarrollar nuevos conceptos que permitan utilizar de manera más concisa las formas que la complejidad organizacional y administrativa contemporánea requiere.

\section{Referencias}

Bhambra, G. K. (2014). Postcolonial and decolonial dialogues. Postcolonial Studies, 17(2), 115-121.

Dussel, E. (2001). Eurocentrismo y modernidad (Introducción a las lecturas de Frankfurt), en W. Mignolo (comp.), Capitalismo y geopolitica del conocimiento. El eurocentrismo y la filosofía de la liberación en el de bate intelectual contemporáneo (pp. 57-70). Buenos Aires: Signos.

Grosfoguel, R. (2007). The epistemic decolonial turn: Beyond political-economy paradigms. Cultural Studies, 21(2-3), 211-223.

Mignolo, W. D. (2010). Desobediencia epistémica. Retórica de la modernidad, lógica de la colonialidad y gramática de la descolonialidad. Buenos Aires: Ediciones del Signo.

Mignolo, W. (2011). The darker side of Western Modernity: Global futures, decolonial options. London: Duke University Press.

Quijano, A. (2009). Colonialidad del Poder y Des/colonialidad del Poder. Conferencia, XXVII Congreso de la Asociación Latinoamericana de Sociología. Buenos Aires, Septiembre. 